\title{
Machine learning of reaction properties via learned representations of the condensed graph of reaction
}

\author{
Esther Heid ${ }^{1}$ and William H. Green ${ }^{1, *}$ \\ ${ }^{1}$ Department of Chemical Engineering, Massachusetts Institute of Technology, Cambridge, Massachusetts 02139, United States
}

\begin{abstract}
The estimation of chemical reaction properties such as activation energies, rates or yields is a central topic of computational chemistry. In contrast to molecular properties, where machine learning approaches such as graph convolutional neural networks (GCNNs) have excelled for a wide variety of tasks, no general and transferable adaptations of GCNNs for reactions have been developed yet. We therefore combined a popular cheminformatics reaction representation, the socalled condensed graph of reaction (CGR), with a recent GCNN architecture to arrive at a versatile, robust and compact deep learning model. The CGR is a superposition of the reactant and product graphs of a chemical reaction, and thus an ideal input for graph-based machine learning approaches. The model learns to create a data-driven, task dependent reaction embedding that does not rely on expert knowledge, similar to current molecular GCNNs. Our approach outperforms current stateof-the-art models in accuracy, is applicable even to imbalanced reactions and possesses excellent predictive capabilities for diverse target properties, such as activation energies, reaction enthalpies, rate constants, yields or reaction classes. We furthermore curated a large set of atom-mapped reactions along with their target properties, which can serve as benchmark datasets for future work. All datasets and the developed reaction GCNN model are available online, free of charge and opensource.
\end{abstract}

\section{INTRODUCTION}

Machine learning models to predict molecular properties have seen a large surge in popularity in the last decade, leading to new developments and impressive performances on the prediction of quantum-mechanical properties ${ }^{1-3}$ biological effects ${ }^{4-6}$ or physicochemical properties,${ }^{7-9}$ to name just a few. In particular, graphbased approaches are on the rise, and have proven both powerful and useful in fields such as drug discovery. ${ }^{10}$

Many representations and model architectures have been developed for the property prediction of molecules. Popular approaches range from conventional machine learning models on fingerprints or descriptors, ${ }^{11}$ graphconvolutional neural networks on $2 \mathrm{D}$ graphs, ${ }^{1,3,8,9}$ and spatial convolutions on $3 \mathrm{D}$ coordinates ${ }^{2,12,13}$ to natural language processing on string representations, ${ }^{14,15}$ amongst others. In contrast, the development of representations and architectures to predict the properties of chemical reactions, i.e. the transformation from one molecule to another, lags behind. Recent studies include the prediction of reaction yields via a random forest model on selected descriptors ${ }^{16}$, a random forest model on structure-based fingerprints ${ }^{17}$ or a molecular transformer model on reaction strings. ${ }^{18}$ Reaction barriers were successfully predicted with both linear regression and neural network models on expert-selected features ${ }^{19}$ or Gaussian Process Regression on selected computational results. ${ }^{20}$ Reaction rates were estimated via deep neural network models on expert features, ${ }^{21}$ as well as selectivities via different models on expertcurated descriptors. ${ }^{22}$ With the notable exception of the seminal work of Schwaller et al. ${ }^{18}$, all these approaches rely on manually created sets of descriptors or features, which hinders the ability to transfer model architectures and representations to new tasks. Recent advances to- wards a more data-driven reaction representation mainly concern the field of retrosynthesis ${ }^{23-26}$ forward reaction prediction, ${ }^{27-32}$ or learning the potential energy surface of a reaction. ${ }^{33}$ Furthermore, a dual graph-convolutional neural network was recently proposed for the prediction of activation energies, but is unable to handle imbalanced reactions. ${ }^{34}$ General architectures which can address a variety of reaction properties are still scarce, mainly due to a lack of a general reaction representation.

Within the field of cheminformatics, the condensed graph of reaction (CGR) ${ }^{35,36}$ which is a superposition of the reactant and product molecules of a reaction, was found to be a suitable reaction representation for a diverse set of tasks. It can be easily constructed from an atom-mapped reaction by assigning dual labels to each bond and atom according to their properties in the reactants and products, respectively. Amongst others, CGRs were successfully used for structure-reactivity modeling, ${ }^{37-39}$ reaction condition prediction, ${ }^{40,41}$ atommapping error identification ${ }^{42}$ and reaction similarity searches. ${ }^{35}$ Toolkits are available to generate or process CGRs, such as the python library CGRTools. ${ }^{43}$ Despite these promising results, the condensed graph of reaction has not been utilized as input representation to more complex models, yet.

In this study, we therefore adapt a graph-convolutional neural network to encode the condensed graph of reaction instead of a molecular graph, and successfully predict reaction properties such as activation energies, reaction enthalpies, rate constants, yields or reaction classes. The developed architecture is general, versatile, and provides a large improvement in accuracy compared to current reaction prediction approaches over a broad field of tasks. 


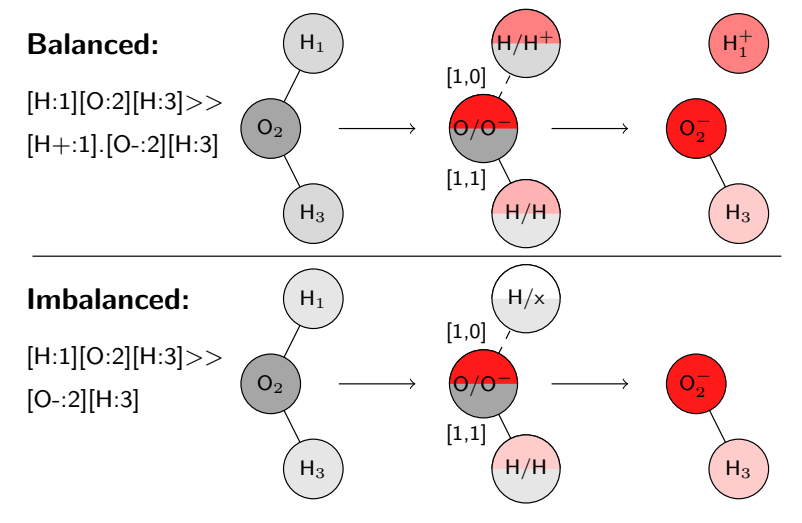

Figure 1. Schematic depiction of the CGR (middle) for the dissociation of water, constructed from the atom-mapped reactants (right) and the atom-mapped products (left). Top: Example of balanced reaction. Bottom: Example of imbalanced reaction. In the CGR, each atom and each bond has two labels, one corresponding to the reactants, another to the products, or a None label (' $\mathrm{x}$ ') for imbalanced reactions.

\section{METHODS}

\section{A. Condensed graph of reaction}

The CGR is a simple superposition of the reactant and product graphs of the molecules in a reaction. The atom mapping of the reaction links the two graphs, and thus provides an important input to correctly construct the CGR. Fig. 1 depicts the atom-mapped reactant molecules in gray (left), as well as the atom-mapped product molecule in red (right) for the dissociation of water. In the middle, the resulting CGR is visualized. The two-colored atoms represent the dual properties of each atom before and after the reaction. The bonds undergoing changes are depicted as dashed lines, and the labels indicate the bond type before and after the reaction. Usually, changes in an atom concern its charge, hybridization, multiplicity or its environment, whereas changes in a bond concern its bond type. ${ }^{43}$ Usually labels that are the same for reactants and products, e.g. $[1,1]$ for the bond from $\mathrm{O}_{2}$ to $\mathrm{H}_{3}$, or $\mathrm{H} / \mathrm{H}$ for $\mathrm{H}_{3}$ are collapsed into a single label, ${ }^{43}$ but we deliberately keep both labels, as each label is used later to construct a part of the atomic and bond features vectors of the CGR graph representation.

\section{B. D-MPNN architecture}

In the following we briefly summarize the architecture of molecular directed message passing neural networks (D-MPNNs), a class of graph-convolutional neural networks (GCNNs), to provide context to the necessary changes and adaptions to generalize from molecules to reactions. We only discuss the directed message passing architecture from Ref. 8, but the described changes can
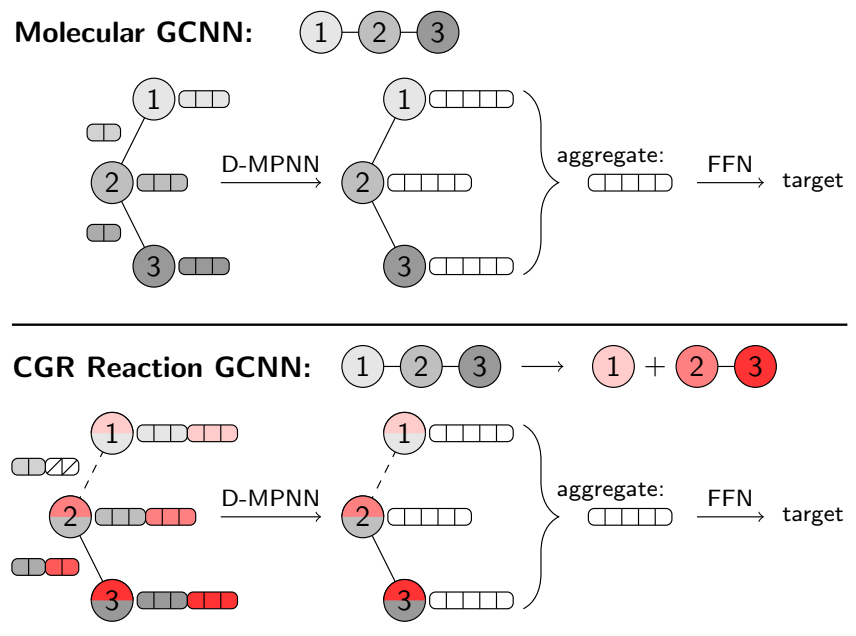

Figure 2. Architecture of a standard graph convolutional neural net (top) and adaption to reactions via input of the condensed graph of reaction (bottom). Each atom and bond fingerprint now consists of two parts, one describing the reactants (gray), the other the products (red). If a bond does not exist in reactants or products, or an atom is missing in an imbalanced reactions, the corresponding parts of the fingerprint (white, crossed out) are set to zero. The white vectors correspond to the hidden atomic and molecular representations.

be easily adapted to any other graph-based architecture.

In general, GCNNs take the graph of a molecule as input, where atoms correspond to vertices in the graph, and bonds to edges. The vertices and edges are usually associated with feature vectors, which describe the identity of an atom, as well as the type of a bond. The vertex or edge features are updated iteratively through exchanging information with their neighbors to create a learned representation of each atom. A representation of the whole molecule is then obtained by an aggregation function, often a simple sum or mean of the atomic representations. The molecular embedding is then passed to a readout function, in most cases a feed-forward neural network $(\mathrm{FFN})$ to relate it to a target property. The whole architecture, i.e. the graph convolutions, aggregation and FFN are usually trained at the same time, end-to-end.

In the case of D-MPNNs, messages are associated with directed edges instead of vertices, in contrast to regular MPNN architectures. The architecture of Yang et al. ${ }^{8}$ is schematically depicted in Fig. 2, top panel. For a molecular graph $G$, initial atom features $\left\{x_{v} \mid v \in V\right\}$ for all vertices $V$ are constructed from a one-hot encoding of the atomic number, degree, formal charge, chirality, number of hydrogens, hybridization and aromaticity of the atom, as well as the scaled atomic mass, resulting in vectors of length 133. Initial bond features $\left\{e_{v w} \mid v w \in E\right\}$ for all edges $E$ describe the bond type, whether the bond is conjugated, in a ring, and contains stereochemical information, resulting in vectors of length 28 . The initial directed edge features $h_{v w}^{0}$ are constructed via appending the features of the first atom of a bond, $x_{v}$ to the respec- 
Table I. Summary of employed datasets.

\begin{tabular}{|c|c|c|c|c|c|c|c|c|c|c|}
\hline Dataset & Datapoints & Ref. & expl. H & bal. & split & task & span & $\mathrm{MAE}_{\text {base }}$ & unit & epochs \\
\hline$E_{a} \omega \mathrm{B} 97 \mathrm{X}-\mathrm{D} 3^{\mathrm{a}}$ & 23,923 & 44 & yes & yes & directed scaffold & regression & 0 to 205 & 25 & $\mathrm{kcal} / \mathrm{mol}$ & 100 \\
\hline$E_{a} \mathrm{E} 2 / \mathrm{S}_{N} 2$ & 3,626 & 45 & yes & yes & random & regression & 0 to 65 & 11 & $\mathrm{kcal} / \mathrm{mol}$ & 100 \\
\hline$E_{a} \mathrm{~S}_{N} \mathrm{Ar}$ & 443 & 20 & no & yes & random & regression & 13 to 42 & 2.9 & $\mathrm{kcal} / \mathrm{mol}$ & 500 \\
\hline$\Delta H$ Rad-6-RE & 63,849 & 46 & yes & yes & directed scaffold & regression & -6 to 12 & 1.8 & $\mathrm{eV}$ & 100 \\
\hline $\log (k)$ Rate constants & 779 & 47 & yes & yes & random & regression & -5 to 10 & 1.9 & unitless & 100 \\
\hline Yield Phosphatases & 33,355 & 48 & no & yes & scaffold & regression & 0 to $1^{\mathrm{b}}$ & 0.10 & unitless & 100 \\
\hline Pistachio & $1,074,765$ & 49 & no & no & random & multiclass class. & $937^{\mathrm{c}}$ & - & - & 30 \\
\hline USPTO-1k-TPL & 445,117 & 50 & no & no & predefined & multiclass class. & $1000^{\mathrm{c}}$ & - & - & 30 \\
\hline
\end{tabular}

${ }^{\text {a }}$ pretraining on 32,731 datapoints at the B97-D3 level of theory

b 4 datapoints have yields higher than 1 due to uncertainties in the assay evaluation.

${ }^{c}$ Number of classes

tive bond features, $e_{v w}$, and passing the concatenated vector to a single neural network layer

$$
h_{v w}^{0}=\tau\left(\mathbf{W}_{i} \operatorname{cat}\left(x_{v}, e_{v w}\right)\right)
$$

with $\mathbf{W}_{i} \in \mathbb{R}^{h \times h_{i}}$ and $h$ being the hidden size (default $300)$, and $h_{i}$ the size of $\operatorname{cat}\left(x_{v}, e_{v w}\right)$, here 147 , and $\tau(\dot{)}$ being a nonlinear activation function. The directed edge features are then updated via an appointed number of message passing steps $t=T$ (default 3 ),

$$
h_{v w}^{t+1}=\tau\left(h_{v w}^{0}+\mathbf{W}_{h} \sum_{k \in\{N(v) \backslash w\}} h_{k v}^{t}\right)
$$

where $\mathbf{W}_{h} \in \mathbb{R}^{h \times h}$ and $N(v) \backslash w$ denotes the neighbors of node $v$ excluding $w$. The hidden states are then transformed back to atom features,

$$
h_{v}=\tau\left(\mathbf{W}_{o} \operatorname{cat}\left(x_{v}, \sum_{w \in N(v)} h_{w v}^{T}\right)\right)
$$

with $\mathbf{W}_{o} \in \mathbb{R}^{h \times h_{o}}$, and $h_{o}$ being the size of $x_{v}$ and $h$. The atomic representations $h_{v}$ can then be aggregated to a molecular feature vector

$$
h=\sum_{v \in G} h_{v}
$$

and optionally augmented with precomputed molecular features $f$ as cat $(h, f)$. The molecular fingerprints are then passed to one or multiple FFN layers.

To adapt the D-MPNN architecture to reactions, two main changes are necessary. First, the list of bonds now encompasses all pairs of atoms that have a bond in either the reactants, or the products, or both, i.e. $E=E^{\text {reac }} \cup E^{\text {prod }}$ of the reactant $G^{\text {reac }}$ and product $G^{\text {prod }}$ graphs. Likewise, the list of atoms comprises all atoms that are present in either reactants, products or both, $V=V^{\text {reac }} \cup V^{\text {prod }}$. Second, the initial atom and bond feature vectors now contain two parts, extracted from the reactant and product graphs separately, one corresponding to the reactants, the other to the products, or the difference between reactants and products. If an atom or bond only occurs on one side of the reaction, its respective feature vector is set to zero on the other side. For atoms, we do not repeat the one-hot encoding of the atomic number, since it cannot change during a chemical reaction, but the scaled mass information is kept for both reactants and products, to not lose isotope information in case of imbalanced reactions. We tested the combination of reactant+product, product+difference(reactantproduct) and reactant+difference(product-reactant) and found that reactant+difference usually performs best. All results reported in this study were obtained with this setting, i.e. $x_{v}=\operatorname{cat}\left(x_{v}^{\text {reac }}, \tilde{x}_{v}^{\text {diff }}\right)$ with length 165 , where the tilde denotes the vector missing the atomic number information, and $e_{v w}=\operatorname{cat}\left(e_{v w}^{\text {reac }}, e_{v w}^{\text {diff }}\right)$ with length 28. All options are available in the provided code on GitHub $^{51}$ and can be tuned as hyperparameters. The bottom panel of Fig. 2 schematically depicts the adapted architecture, where the gray parts of the initial fingerprints correspond to the reactants, and the red parts to the products. The two changes thus only concern the creation of the graph object, as well as the initialization of the edge and vertex features. The remaining parts of the model, i.e. Eq. (1)-(4), are unchanged.

\section{Data preparation}

We utilized four reaction databases from literature as provided, as well as cleaned and atom-mapped four more, which we made openly available on GitHub. ${ }^{52}$ Table I provides a compact overview over all employed datasets.

1. Computational activation energies of forward and reverse reactions at the $\omega$ B97X-D3/def2-TZVP level of theory (as well as at the B97-D3/def2mSVP level of theory for pretraining) were used as provided in Ref. 44. The dataset features a diverse set of reactions transforming unimolecular reactants into uni- or multi-molecular products, and is already atom-mapped.

2. Computational activation energies for competing $\mathrm{E} 2 / \mathrm{S}_{N} 2$ were furthermore taken from Ref 45 , and 
atom-mapped manually using heuristic substitution patterns. The resulting database is published along with this study.

3. Experimental activation energies for $\mathrm{S}_{\mathrm{N}} \mathrm{Ar}$ reactions were taken as provided from Ref. 20. All reactions were already atom-mapped, and furthermore contained information about the solvent each reaction was carried out in, as well as the computational activation energy at the $\omega \mathrm{B} 97 \mathrm{X}-\mathrm{D} / 6-311+\mathrm{G}(\mathrm{d}, \mathrm{p})$ level of theory. The solvent descriptors and computational activation energies were passed to the model as molecular fingerprints $f$.

4. Computational reaction enthalpies were taken from the Rad-6-RE database ${ }^{46}$ and atom-mapped via Grzybowski's algorithm. ${ }^{53}$ Imbalanced reactions were discarded, since Ref. 46 claims to only report balanced reactions. We thus assumed that imbalanced reaction correspond to an error. Both forward and reverse reactions were taken into account. The resulting database is published along with this study. We note that reaction enthalpies could also be modeled via training a single model to predict molecular enthalpies, ${ }^{54,55}$ and converting the enthalpies of reactants and products into the respective enthalpies of reaction. This approachs was followed by Stocker et al. ${ }^{46}$ however, in this work we instead want to highlight the direct prediction of reaction enthalpies.

5. Reaction rate constants were taken from Ref. 47 and atom-mapped via Grzybowski's algorithm. ${ }^{53}$ Models were then trained on the logarithm of the rate constants at $1000 \mathrm{~K}, \log \left(\frac{k(1000 K)}{k_{\text {ref }}}\right)$, with $k$ in $\mathrm{cm}^{3} \mathrm{~mol}^{-1} \mathrm{~s}^{-1}$ (bimolecular) or $\mathrm{s}^{-1}$ (unimolecular) depending on the reaction mechanism, and $k_{r e f}=$ 1 in the same units. The resulting database is published along with this study.

6. Experimental reaction yields for 218 phosphatase enzyme sequences on 157 substrates were extracted from Ref. 48. The original article features 167 substrates, but only substrates that contained a single phosphate group were kept. Since the reaction outcomes were not reported in Ref. 48, the product for multi-phosphate substrates are not known with certainty, and were thus not included. The different enzymes were represented as simple onehot encoding and passed to the model as molecular fingerprints $f$. Products and the respective atommappings were calculated manually with a simple set of heuristic rules. The resulting database is published along with this study.

7. The reaction names of one million reactions from an in-house preprocessed and cleaned version of Pistachio $^{49}$ (processing analogous to Ref. 56) were taken with atom-mappings as provided. Since Pistachio is not open-source, the resulting database is not published along with this study.

8. The reaction names of the atom-mapped USPTO$1 \mathrm{k}-\mathrm{TPL}$ dataset recently curated by Schwaller et $a l .{ }^{50}$ were used as-is.

\section{Dummy baselines}

The mean absolute error of a dummy baseline model predicting the mean of the target values in each dataset is given in Table I. Comparing against such a simple baseline helps to judge the quality of a predictive model, where low errors on a dataset with narrow target range can otherwise be mistaken for a satisfactory performance.

\section{E. Other Baselines}

We furthermore examined more complex baseline models. First, the dual GCNN model of Grambow et al. ${ }^{34}$ was trained with hyperparameters similar to the CGR GCNN approach (MPNN depth of 3, hidden size of 300, one FFN layer, no dropout) on all datasets comprising balanced reactions, termed 'Grambow' in the following. We note that the model does not accept imbalanced reactions as input, so that no baseline could be computed for the imbalanced datasets in Table I.

Second, the recently developed BERT deep learning reaction fingerprints ${ }^{50}$ were utilized as input to a regular FFN, where we used a default hidden size of 300 and two FFN layers. The fingerprints were computed using the open-access rxnfp software on non-atom-mapped reaction SMILES. ${ }^{57}$

Third, Morgan fingerprints ${ }^{58}$ were calculated for the reactants and products separately, and either subtracted ('Morgan Diff') or concatenated ('Morgan Concat'), and again served as input to an FFN of hidden size 300 and two FFN layers. Morgan fingerprints at radius 3 and length 1024 were calculated via RDKit. ${ }^{59}$

We furthermore tried to utilize ISIDA descriptors ${ }^{35,43}$ as inputs to an FFN, but found the calculation to be computationally infeasible for larger datasets, since the descriptors extract a dataset-dependent set of reaction fragments from all available reactions, and thus need to hold the full set of reactions and all their fragments in memory at the same time.

\section{F. Model parameters}

A hyperparameter search for the optimal hidden size, number of layers, number of message passing steps and dropout rate was computed via 20 steps of Bayesian Optimization for the CGR GCNN, Grambow's dual GCNN and all fingerprint models as implemented in 
Table II. Comparison of performances (mean absolute errors) and the respective number of trainable parameters of regression tasks between the CGR graph convolutional model of this study, Grambow's dual GCNN of Ref. ${ }^{34}$ and the best performing FFN on reaction fingerprints. Intervals correspond to the mean and standard deviation of five folds. Best performance per dataset highlighted in bold.

\begin{tabular}{llccccc} 
Dataset & unit & CGR default & CGR opt & Grambow default & Grambow opt & best FP opt \\
\hline \hline Model performance: & & & & & & \\
$E_{a} \omega \mathrm{B} 97 \mathrm{X}-\mathrm{D} 3$ (pretr. B97-D3) & $\mathrm{kcal} / \mathrm{mol}$ & $4.84 \pm 0.29$ & $\mathbf{4 . 2 5} \pm \mathbf{0 . 1 9}$ & $6.35 \pm 0.26$ & $5.26 \pm 0.15$ & $10.94 \pm 0.29$ \\
$E_{a} \mathrm{E} 2 / \mathrm{S}_{N} 2$ & $\mathrm{kcal} / \mathrm{mol}$ & $\mathbf{2 . 6 4} \pm \mathbf{0 . 1 0}$ & $2.65 \pm 0.09$ & $2.76 \pm 0.08$ & $2.86 \pm 0.07$ & $3.37 \pm 0.10$ \\
$E_{a} \mathrm{~S}_{N} \mathrm{Ar}$ & $\mathrm{kcal} / \mathrm{mol}$ & $0.86 \pm 0.12$ & $\mathbf{0 . 8 6} \pm \mathbf{0 . 0 9}$ & $1.09 \pm 0.20$ & $0.95 \pm 0.23$ & $1.11 \pm 0.13$ \\
$\Delta H$ Rad-6-RE & $\mathrm{eV}$ & $0.16 \pm 0.01$ & $\mathbf{0 . 1 3} \pm \mathbf{0 . 0 1}$ & $0.40 \pm 0.01$ & $\mathbf{0 . 0 7}$ to $\mathbf{0 . 4 3} \mathbf{3}^{\mathrm{a}}$ & $0.64 \pm 0.01$ \\
$\log (k)$ Rate constants & unitless & $\mathbf{0 . 4 1} \pm \mathbf{0 . 0 5}$ & $0.41 \pm 0.02$ & $0.60 \pm 0.05$ & $0.45 \pm 0.04$ & $0.66 \pm 0.08$ \\
Yield Phosphatases & unitless & $0.063 \pm 0.002$ & $\mathbf{0 . 0 6 2} \pm \mathbf{0 . 0 0 1}$ & $0.081 \pm 0.002$ & $0.068 \pm 0.003$ & $0.063 \pm 0.004$ \\
\hline
\end{tabular}

\section{Model size:}

$E_{a} \omega$ B97X-D3 (pretr. B97-D3)

$E_{a} \mathrm{E} 2 / \mathrm{S}_{N} 2$

$E_{a} \mathrm{~S}_{N} \mathrm{Ar}$

$\Delta H$ Rad-6-RE

$\log (k)$ Reaction rates

Yield Phosphatases
378,601

378,601

380,401

378,601

378,601

444,001

$\begin{array}{cc}10,371,601 & 361,801 \\ 2,817,101 & 361,801 \\ 6,395,201 & 361,807 \\ 10,371,601 & 361,801 \\ 6,393,701 & 361,801 \\ 6,692,001 & 362,019\end{array}$

$24,877,601$

$6,381,601$

$16,754,401$

361,201

$8,278,201$

79,201

$20,035,401$

$8,269,801$

$6,381,601$

$11,080,001$

a See SI for details on the Rad-6-RE model performance

Chemprop. ${ }^{8}$ Optimized models are termed 'opt' throughout this study. More details are given in the Supporting Information. All models were trained with a batch size of 50, ReLU activation functions, mean aggregation between the MPNN and FFN step, and explicit hydrogens as specified in Table I. Learning rates were increased linearly from $10^{-4}$ to $10^{-3}$ for two epochs, and then decreased exponentially from $10^{-3}$ to $10^{-4}$. Prior to hyperparameter optimization, no dropout, three iterations of message passing and a hidden size of 300 were used (termed 'default'). Regression models used mean absolute error as the metric for evaluation and early stopping; classification models instead used accuracy as metric. All models were trained on five different data splits to arrive at a split-independent estimate of the true model performance. Split sizes of $80 / 10 / 10$ for training, validation and test set, were used if not indicated otherwise. Table I lists the split types for each dataset. Scaffold splits were performed on the reactant side of the $E_{a} \omega \mathrm{B} 97 \mathrm{X}-$ D3, $\Delta H$ Rad-6-RE and Yield Phosphatases databases, where multiple molecular scaffolds were identified. Both the $E_{a} \omega \mathrm{B} 97 \mathrm{X}-\mathrm{D} 3$ and the $\Delta H$ Rad-6-RE datasets comprise forward and reverse reactions, so that special care was taken to enforce that each pair of forward and reverse reactions was placed in the same set. Otherwise, the test set error of a model is unrealistically low, and does not reflect the true model performance. Random splits were performed on the $E_{a} \mathrm{E} 2 / \mathrm{S}_{N} 2, E_{a} \mathrm{~S}_{N} \mathrm{Ar}$ and $\log (k)$ Rate constants databases since they consisted of too few scaffolds to perform a meaningful scaffold split. A random split was furthermore performed on the Pistachio dataset. For the USPTO-1k-TPL dataset, the split into training and test data was taken from Ref. 50, and the training set was split into a training and validation set randomly.

\section{RESULTS AND DISCUSSION}

Table II summarizes the performances of the CGR GCNN developed in this study, Grambow's dual $\mathrm{GCNN}^{34}$ and the best performing fingerprint model (FFN on either the Bert, Morgan Diff or Morgan Concat fingerprints). A full list of test performance of all default and optimized models on all tasks is available in the Supporting Information. The CGR approach outperforms all other models both with its default hyperparameters, as well as after hyperparameter optimization for all datasets. We also attempted to make comparisons to the reaction data presented in Ref. 46 ( $\Delta H$ Rad-6$\mathrm{RE}$ ), but for technical reasons discussed in the SI it is difficult to fairly compare the methods on this particular dataset. In all systems, the default hyperparameters are close to the ideal hyperparameters, indicating that even the small, compact default model is able to learn complex target properties. In the following, we analyze the performances on each target in detail.

\section{A. Prediction of activation energies}

The performance of the CGR model for the prediction of computational and experimental activation energies was evaluated on three different datasets. The first dataset, $E_{a} \omega \mathrm{B} 97 \mathrm{X}-\mathrm{D} 3$, is by far the largest and most diverse dataset, comprising about 24,000 computational activation energies for various elemental reactions in the forward and reverse direction. Its wide range of target values (0 to $205 \mathrm{kcal} / \mathrm{mol}$ ) makes an accurate prediction extremely challenging, so that we consider the observed lowest errors of about $4 \mathrm{kcal} / \mathrm{mol}$ a success nevertheless. For comparison, a model predicting the mean of the dataset for each datapoint would possess a mean 


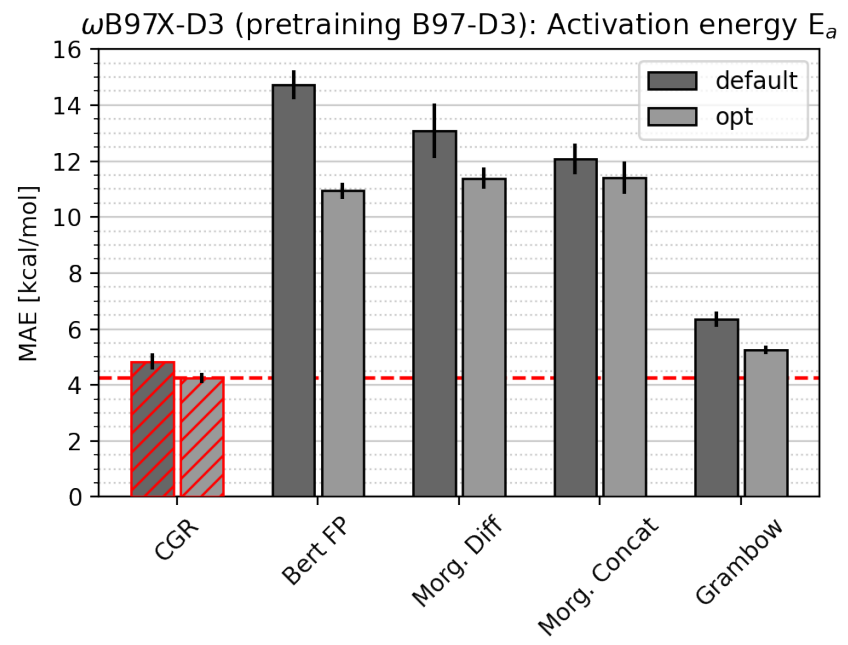

Figure 3. Comparison of test set mean absolute error between different models for the $\omega$ B97X-D3 computational activation energy dataset, with pretraining on B97-D3 activation energies. Error bars correspond to the standard deviation between five folds. Best model system highlighted in red, line corresponds to best performance.

absolute error of about $25 \mathrm{kcal} / \mathrm{mol}$. Fig. 3 depicts the performance of various default and optimized architectures, where the CGR model clearly outperforms other models. All fingerprint models perform very poorly, highlighting the inability of reaction fingerprints to encode certain details of a transformation especially for diverse datasets, even despite the large sizes of some of the optimized models. We furthermore note that the obtained performance of the dual GCNN model differs from the results in Ref. 34 due to the different, more rigorous data splits used in this study. As mentioned in the previous section, placing forward and reverse reactions in different data splits, so that some of the reactions in the test set also appear in the training set (but in reverse direction) can severely overestimate model performance. The errors reported in Table II and Fig. 3 thus provide a more accurate estimation of the the true predictive power of Grambow's dual-GCNN model than the numbers reported in Ref. 34.

The second dataset, $E_{a} \mathrm{E} 2 / \mathrm{S}_{N} 2$, only comprises two chemical transformations, namely E2 and $\mathrm{S}_{N} 2$ of different electrophiles and nucleophiles. It spans computational activation energies of $0-65 \mathrm{kcal} / \mathrm{mol}$, and possesses only a few thousand datapoints. The baseline performance of a model predicting the mean of the dataset for each datapoint is about $11 \mathrm{kcal} / \mathrm{mol}$. This reduction in target range and chemistry helps all models to perform better, as depicted in Fig. 4. Again, the CGR approach outperforms all other models, but by a smaller margin. Also, the fingerprint models feature a comparatively better performance than with the previous dataset, since the possible chemical transformations are very few, and differences in the activation energies can be related to the

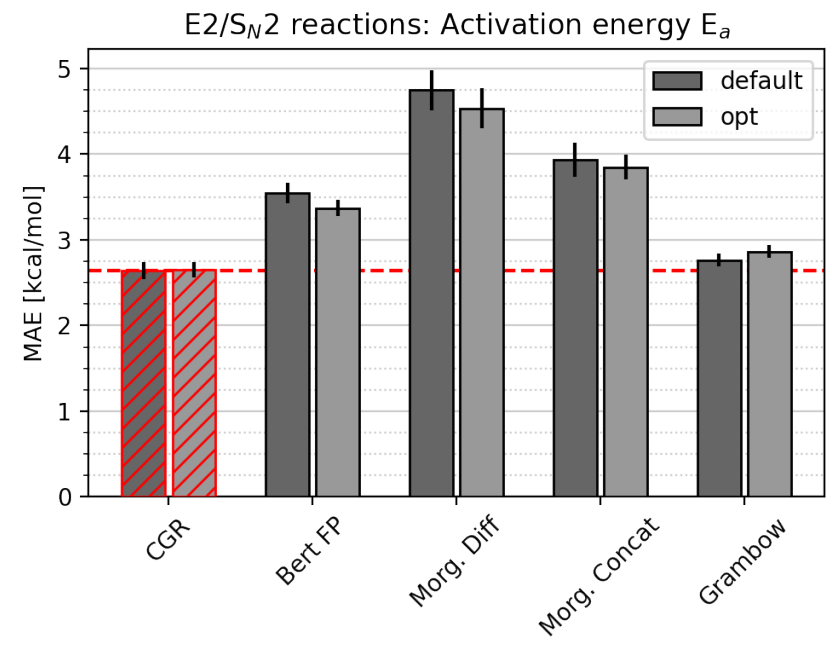

Figure 4. Comparison of test set mean absolute error between different models for the E2/ $\mathrm{S}_{N} 2$ computational activation energy dataset. Error bars correspond to the standard deviation between five folds. Best model system highlighted in red, line corresponds to best performance.

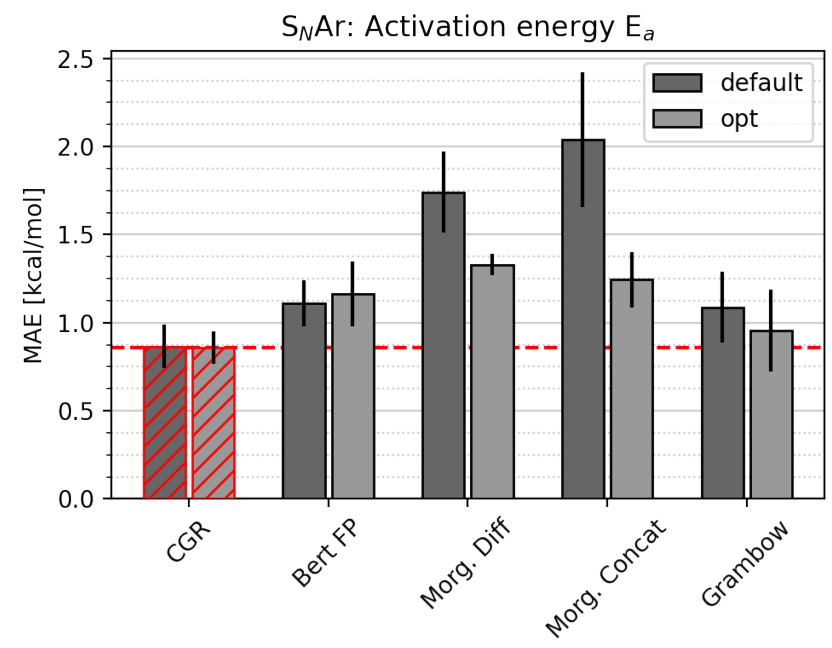

Figure 5. Comparison of test set mean absolute error between different models for the $\mathrm{S}_{N} \mathrm{Ar}$ experimental activation energy dataset. Error bars correspond to the standard deviation between five folds. Best model system highlighted in red, line corresponds to best performance.

fingerprints of reactants and products more straightforwardly.

The third dataset, $E_{a} \mathrm{~S}_{N} \mathrm{Ar}$, is different from the first two datasets in three regards. First, it is very small, comprising only a few hundred reactions. Second, it is very narrow, spanning only values between $13-42 \mathrm{kcal} / \mathrm{mol}$, which enables even a simple baseline model predicting only the mean of the distribution to perform, seemingly, well with a mean absolute error of about $3 \mathrm{kcal} / \mathrm{mol}$. Third, additional input beyond the 


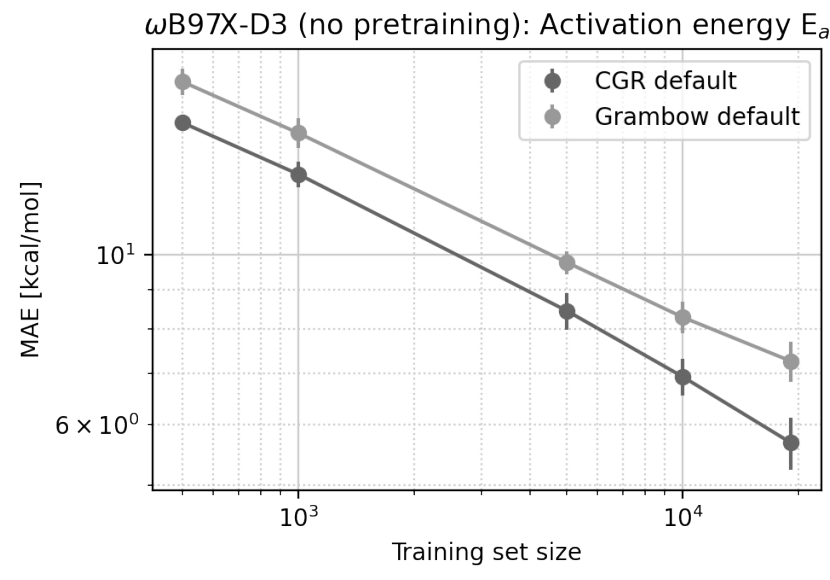

Figure 6. Mean absolute errors of the CGR GCNN model on subsets of the $E_{a} \omega \mathrm{B} 97 \mathrm{X}-\mathrm{D} 3$ dataset without pretraining.

reaction itself is provided, namely five solvent descriptors to characterize the employed solvent and the computational activation energy. Fig. 5 depicts the performance of all studied models, where the CGR approach leads to the lowest mean absolute errors, but is not significantly better than the optimized Grambow dual GCNN model. In literature, Gaussian Process Regression on a large set of quantum-mechanically derived descriptors for this dataset yielded a mean absolute error of $0.77 \mathrm{kcal} / \mathrm{mol} .{ }^{20}$ The CGR GCNN approach comes reasonably close to this benchmark, taking into account that it only learns from the reaction graphs, and does not feature any quantum-mechanical descriptors apart from the solvent information and the computed Ea's. The requirement for quantum-mechanical descriptors as input can greatly increase the computer time required to make a prediction, but it may be possible to avoid this by building a model for predicting the quantum-mechanical descriptors as we done recently by Guan et al. ${ }^{60}$

A comparison of the performance of the CGR architecture to the dummy baselines across the three datasets yields another interesting insight. Even with very little data $\left(E_{a} \mathrm{~S}_{N} \mathrm{Ar}\right)$ the CGR model can still produce a relatively low MAE, at approximately a third of the error of the dummy model. Adding more data, the MAE decreases to a fourth of the dummy model MAE $\left(E_{a}\right.$ $\left.\mathrm{E} 2 / \mathrm{S}_{N} 2\right)$, or even a sixth $\left(E_{a} \omega \mathrm{B} 97 \mathrm{X}-\mathrm{D} 3\right)$, with further reduction expected for more datapoints. An evaluation of model performance with training set size for the $E_{a}$ $\omega$ B97X-D3 dataset without pretraining is shown in Fig. 6 for the default CGR and dual GCNN models. The CGR GCNN model performance does not level off, indicating that the model may achieve chemical accuracy if a sufficiently large dataset was provided. A simple extrapolation predicts the model to achieve chemical accuracy with 5-10 million datapoints, which is not out of reach in light of the current advances in high-performance computing. In contrast, the dual GCNN model levels off

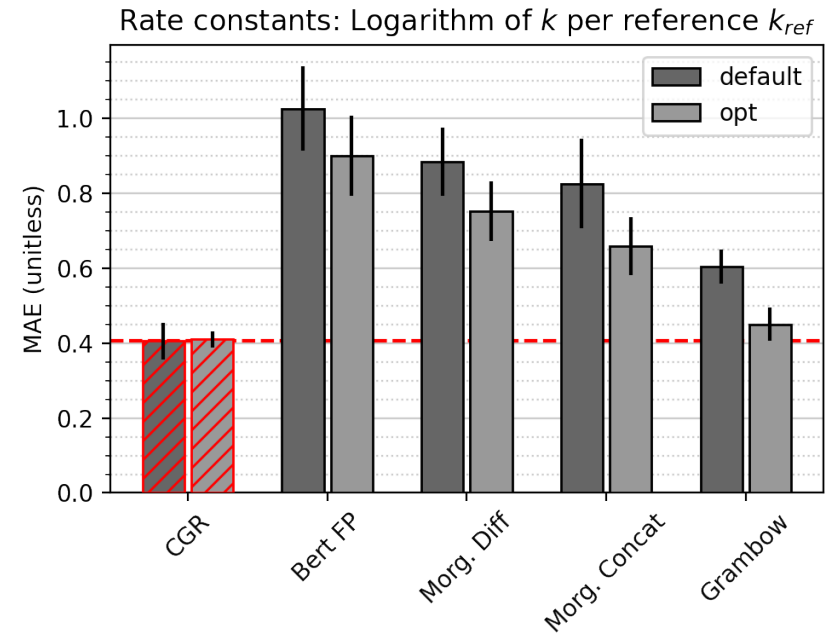

Figure 7. Comparison of test set mean absolute error between different models for the computational rate constants dataset. Error bars correspond to the standard deviation between five folds. Best model system highlighted in red, line corresponds to best performance.

slightly, and even if linear behavior is assumed, would only reach chemical accuracy at 100-300 million datapoints.

\section{B. Prediction of rate constants}

Model performances for predicting rate constants (at $1000 \mathrm{~K}$ ) are shown in Fig. 7, where again the CGR GCNN outperforms other approaches. We note that the errors are reported for the logarithm of the rate constant, so that an MAE of 0.4 corresponds to deviations of about $2.5 \mathrm{~cm}^{3} \mathrm{~mol}^{-1} \mathrm{~s}^{-1}$ (bimolecular) or $\mathrm{s}^{-1}$ (unimolecular). This is well within or even below the accuracy of the rates at the employed level of theory, M06-2X/MG3S (compared to more elaborate computational results utilizing CCSD(T)-F12/RI calculations with the cc-VTZF1256 and cc-VTZ-F12-CABS57 basis sets, see Ref. 47).

\section{Prediction of reaction yields}

A different picture arises for the prediction of reaction yields, Fig. 8. All models perform about equally well, and are only slightly better than a dummy baseline model predicting the mean of the distribution (with an MAE of 0.10 , see Table I). The CGR approach outperforms other models by a slight margin, but overall, all model performances are rather mediocre. Since the dataset contains only 157 substrates in combination with 218 enzymes, and the enzymes were merely one-hot-encoded, the subprime performance is not surprising. In other words, the models can pick up relations for the different substrates 


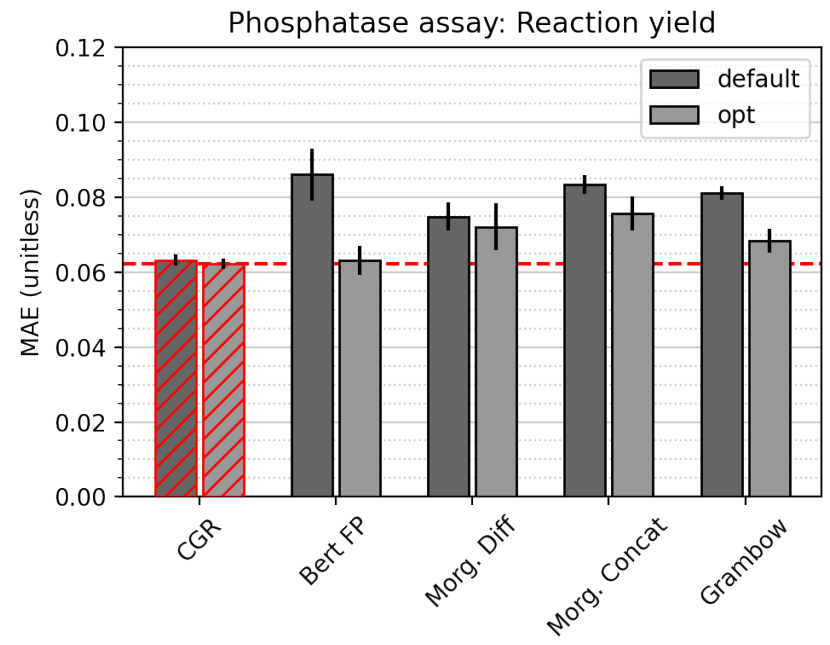

Figure 8. Comparison of test set mean absolute error between different models for the experimental phosphatase reaction yield dataset. Error bars correspond to the standard deviation between five folds. Best model system highlighted in red, line corresponds to best performance.

well, but can still be hampered by the crude encoding of the protein information.

\section{Prediction of reaction classes}

We furthermore explored the performance of the CGR GCNN approach on classification tasks, here the classification of reactions into their respective name reactions. To this aim, we predict the names of reactions of two datasets, a preprocessed and cleaned version of Pistachio containing 937 class names, as well as a recently published benchmark, USPTO-1k-TPL, containing 1000 class names. Fig. 9 depicts the top-1-accuracy (fraction of test reactions were the correct name is ranked highest) and top-3-accuracy (fraction of test reactions where the correct name is found within the three highest ranked predictions), depending on the size of the training set. Since the reactions in both datasets are not balanced (leaving groups are not reported on the product side), the performance of Grambow's dual GCNN approach could not be evaluated. We instead compare the observed accuracy to a recent benchmark of Schwaller et al. (red line in Fig. 9), who achieved a 98.9\% top-1-accuracy on USPTO $1 \mathrm{k}$ TPL with their state-of-the-art Transformer model. ${ }^{50}$ They furthermore report $98.2 \%$ accuracy on Pistachio name reactions, but preprocessed and cleaned the data differently, so that no direct comparison is possible. We note that the reaction input to the Transformer model does not rely on atom-mapping, so that the model learns from less information. The CGR approach outperforms the Transformer model, but due to the differences in representation (no atom-mapping vs. atom-mapping), a di-
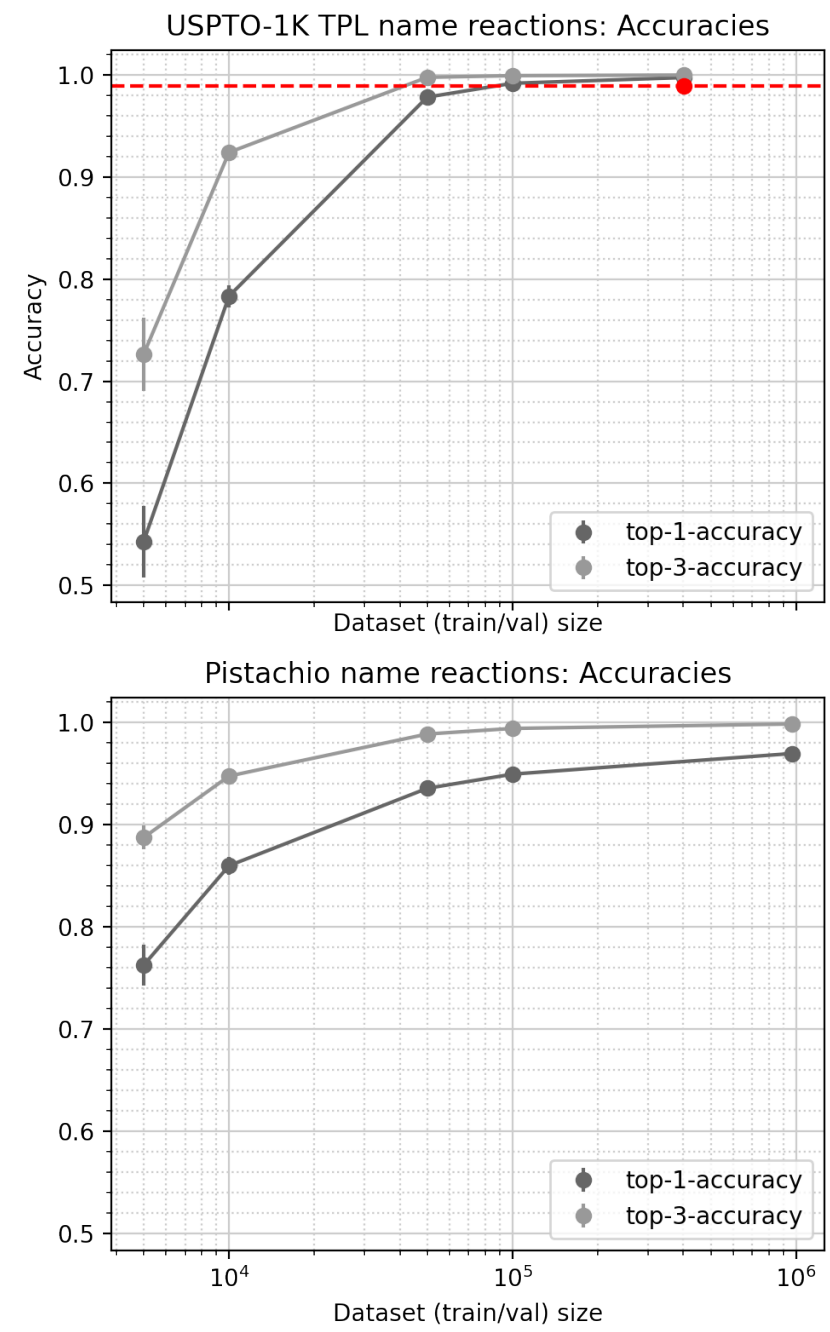

Figure 9. Comparison of accuracies between different models for the classification of name reactions via the USPTO-1KTPL dataset (top) or the Pistachio dataset (bottom). Error bars correspond to the standard deviation between five folds. The red dot and line correspond to the performance achieved by Ref. 50 .

rect comparison is somewhat biased. Nevertheless, the observed accuracies of the CGR GCNN model indicate that it can learn to predict name reactions easily, and that imbalanced reactions do not hamper model training.

\section{E. Limitations}

The CGR GCNN approach developed in this study thus provides a high-performing and flexible alternative to other architectures, such as dual GCNN and FFNs on various fingerprints. It is more flexible than the dual GCNN model in that it can treat imbalanced reactions. However, like the dual GCNN architecture it relies on 
correct atom mapping of reactions, which increases the workload on pre-processing steps of databases significantly. Incorrect atom mappings add noise to the data, so that the quality of a prediction depends to some extent on the quality of the atom-mapping of both training and test data.

\section{CONCLUSION}

We have introduced, benchmarked and validated the use of CGRs as a suitable reaction representation to graph-convolutional neural nets. The resulting CGR GCNNs outperform other current approaches on a wide variety of datasets and prediction tasks. Furthermore, they perform well with a very limited model size, allowing for rapid training and evaluation. We could thus successfully extend the use of GCNNs from molecules to reactions, creating small and convenient models for the prediction of various reaction properties. We expect the developed representation and architecture, as well as the atom-mapped datasets made available along with this article, to seed further developments in the emerging field of reaction property prediction.

\section{DATA AND SOFTWARE AVAILABILITY}

The CGR GCNN model architecture is available on GitHub on the master branch of Chemprop. ${ }^{51}$ Datasets
1, 3 and 8 are available from literature, ${ }^{20,44,50}$ and were used as provided. Datasets 2, 4, 5, and 6 are available on GitHub. ${ }^{52}$ Dataset 7 is proprietary and thus not freely available, but does not provide an integral part of this study, since it only complements dataset 8. For all datasets except 7 we furthermore provide the data splits used in this study, as well as the trained CGR GCNN default models, along with instructions on how to create predictions. $^{52}$

\section{SUPPORTING INFORMATION}

Model performances on the Rad-6-RE database and detailed discussion of the influence of data leakage in this system. Details on hyperparameter searches, full list of test set errors for all models with and without hyperparameter optimization.

\section{ACKNOWLEDGEMENT}

E.H. acknowledges support from the Austrian Science Fund (FWF), project J-4415. The authors acknowledge the Machine Learning for Pharmaceutical Discovery and Synthesis Consortium (MLPDS) for funding. Parts of the data reported within this paper were generated on the MIT SuperCloud Lincoln Laboratory Supercomputing Center. Furthermore, Charles McGill is gratefully acknowledged for helpful discussions and insights.
* whgreen@mit.edu

1 J. Gilmer, S. S. Schoenholz, P. F. Riley, O. Vinyals, and G. E. Dahl, in International conference on machine learning (PMLR, 2017) pp. 1263-1272.

2 J. Klicpera, J. Groß, and S. Günnemann, arXiv preprint arXiv:2003.03123 (2020).

3 S. Zhang, Y. Liu, and L. Xie, arXiv preprint arXiv:2011.07457 (2020).

4 Z. Alperstein, A. Cherkasov, and J. T. Rolfe, arXiv preprint arXiv:1905.13343 (2019).

5 M. Zaslavskiy, S. Jégou, E. W. Tramel, and G. Wainrib, Comp. Toxicol. 10, 81 (2019).

6 P. Li, Y. Li, C.-Y. Hsieh, S. Zhang, X. Liu, H. Liu, S. Song, and X. Yao, Brief. Bioinf. 22, bbaa266 (2021).

7 X. Wang, Z. Li, M. Jiang, S. Wang, S. Zhang, and Z. Wei, J. Chem. Inf. Model. 59, 3817 (2019).

${ }^{8}$ K. Yang, K. Swanson, W. Jin, C. Coley, P. Eiden, H. Gao, A. Guzman-Perez, T. Hopper, B. Kelley, M. Mathea, et al., J. Chem. Inf. Model. 59, 3370 (2019).

9 Z. Wu, B. Ramsundar, E. N. Feinberg, J. Gomes, C. Geniesse, A. S. Pappu, K. Leswing, and V. Pande, Chem. Sci. 9, 513 (2018).

10 O. Wieder, S. Kohlbacher, M. Kuenemann, A. Garon, P. Ducrot, T. Seidel, and T. Langer, Drug Disc. Today: Technol. (2020).
11 A. Capecchi, D. Probst, and J.-L. Reymond, J. Cheminfo. 12, 1 (2020).

12 K. T. Schütt, H. E. Sauceda, P.-J. Kindermans, A. Tkatchenko, and K.-R. Müller, J. Chem. Phys. 148, 241722 (2018).

13 J. Westermayr, M. Gastegger, and P. Marquetand, J. Phys. Chem. Lett. 11, 3828 (2020).

14 S. Jaeger, S. Fulle, and S. Turk, J. Chem. Inf. Model. 58, 27 (2018).

15 Y.-F. Zhang, X. Wang, A. C. Kaushik, Y. Chu, X. Shan, M.-Z. Zhao, Q. Xu, and D.-Q. Wei, Front. Chem. 7, 895 (2020).

16 D. T. Ahneman, J. G. Estrada, S. Lin, S. D. Dreher, and A. G. Doyle, Science 360, 186 (2018).

17 F. Sandfort, F. Strieth-Kalthoff, M. Kühnemund, C. Beecks, and F. Glorius, Chem 6, 1379 (2020).

18 P. Schwaller, A. C. Vaucher, T. Laino, and J.-L. Reymond, Mach. Learn.: Sci. Technol. 2, 015016 (2021).

19 A. R. Singh, B. A. Rohr, J. A. Gauthier, and J. K. Nørskov, Catal. Lett. 149, 2347 (2019).

${ }^{20}$ K. Jorner, T. Brinck, P.-O. Norrby, and D. Buttar, Chem. Sci. 12, 1163 (2021).

21 E. Komp and S. Valleau, J. Phys. Chem. A 124, 8607 (2020).

22 A. F. Zahrt, J. J. Henle, B. T. Rose, Y. Wang, W. T. Darrow, and S. E. Denmark, Science 363 (2019). 
${ }^{23}$ M. H. Segler, M. Preuss, and M. P. Waller, Nature 555, 604 (2018).

${ }^{24}$ P. Schwaller, R. Petraglia, V. Zullo, V. H. Nair, R. A. Haeuselmann, R. Pisoni, C. Bekas, A. Iuliano, and T. Laino, arXiv preprint arXiv:1910.08036 (2019).

25 Q. Yang, V. Sresht, P. Bolgar, X. Hou, J. L. Klug-McLeod, C. R. Butler, et al., Chem. Commun. 55, 12152 (2019).

26 B. Chen, T. Shen, T. S. Jaakkola, and R. Barzilay, arXiv preprint arXiv:1910.09688 (2019).

27 M. A. Kayala and P. Baldi, J. Chem. Inf. Model. 52, 2526 (2012).

28 D. Fooshee, A. Mood, E. Gutman, M. Tavakoli, G. Urban, F. Liu, N. Huynh, D. Van Vranken, and P. Baldi, Mol. Syst. Des. Eng. 3, 442 (2018).

29 J. N. Wei, D. Duvenaud, and A. Aspuru-Guzik, ACS Cent. Sci. 2, 725 (2016).

30 P. Schwaller, T. Laino, T. Gaudin, P. Bolgar, C. A. Hunter, C. Bekas, and A. A. Lee, ACS Cent. Sci. 5, 1572 (2019).

31 B. Liu, B. Ramsundar, P. Kawthekar, J. Shi, J. Gomes, Q. Luu Nguyen, S. Ho, J. Sloane, P. Wender, and V. Pande, ACS Cent. Sci. 3, 1103 (2017).

32 C. W. Coley, W. Jin, L. Rogers, T. F. Jamison, T. S. Jaakkola, W. H. Green, R. Barzilay, and K. F. Jensen, Chem. Sci. 10, 370 (2019).

33 M. Meuwly, Chem. Rev. (2021).

34 C. A. Grambow, L. Pattanaik, and W. H. Green, J. Phys. Chem. Lett. 11, 2992 (2020).

35 A. Varnek, D. Fourches, F. Hoonakker, and V. P. Solov'ev, J. Comput. Aided Mol. Des. 19, 693 (2005).

${ }^{36}$ F. Hoonakker, N. Lachiche, A. Varnek, and A. Wagner, Int. J. Artif. Intell. Tools 20, 253 (2011).

37 T. Madzhidov, P. Polishchuk, R. Nugmanov, A. Bodrov, A. Lin, I. Baskin, A. Varnek, and I. Antipin, Russ. J. Org. Chem. 50, 459 (2014).

38 T. Madzhidov, T. Gimadiev, D. Malakhova, R. Nugmanov, I. Baskin, I. Antipin, and A. Varnek, J. Struct. Chem. 58, 650 (2017).

39 T. Gimadiev, T. I. Madzhidov, R. I. Nugmanov, I. I. Baskin, I. S. Antipin, and A. Varnek, J. Comput. Aid. Mol. Des. 32, 401 (2018).

40 A. I. Lin, T. I. Madzhidov, O. Klimchuk, R. I. Nugmanov, I. S. Antipin, and A. Varnek, J. Chem. Inf. Model. 56, 2140 (2016).

41 G. Marcou, J. Aires de Sousa, D. A. Latino, A. de Luca, D. Horvath, V. Rietsch, and A. Varnek, J. Chem. Inf. Model. 55, 239 (2015).
42 C. Muller, G. Marcou, D. Horvath, J. Aires-de Sousa, and A. Varnek, J. Chem. Inf. Model. 52, 3116 (2012).

43 R. I. Nugmanov, R. N. Mukhametgaleev, T. Akhmetshin, T. R. Gimadiev, V. A. Afonina, T. I. Madzhidov, and A. Varnek, J. Chem. Inf. Model. 59, 2516 (2019).

${ }^{44}$ C. A. Grambow, L. Pattanaik, and W. H. Green, Sci. Data 7, 1 (2020)

45 G. F. von Rudorff, S. N. Heinen, M. Bragato, and O. A. von Lilienfeld, Mach. Learn.: Sci. Technol. 1, 045026 (2020).

46 S. Stocker, G. Csányi, K. Reuter, and J. T. Margraf, Nat. Commun. 11, 1 (2020).

47 P. L. Bhoorasingh, B. L. Slakman, F. Seyedzadeh Khanshan, J. Y. Cain, and R. H. West, J. Phys. Chem. A 121, 6896 (2017).

48 H. Huang, C. Pandya, C. Liu, N. F. Al-Obaidi, M. Wang, L. Zheng, S. T. Keating, M. Aono, J. D. Love, B. Evans, et al., Proc. Nat. Acad. Sci. 112, E1974 (2015).

49 (accessed: 08/2020), Pistachio (Nextmove Software) https://www.nextmovesoftware.com/pistachio.html.

${ }^{50}$ P. Schwaller, D. Probst, A. C. Vaucher, V. H. Nair, D. Kreutter, T. Laino, and J.-L. Reymond, Nat. Mach. Intell. 3, 144 (2021).

51 "Chemprop software," (2021), https://github.com/ chemprop/chemprop.

52 "CSV files of datasets," (2021), https://github.com/ hesther/reactiondatabase.

53 W. Jaworski, S. Szymkuć, B. Mikulak-Klucznik, K. Piecuch, T. Klucznik, M. Kaźmierowski, J. Rydzewski, A. Gambin, and B. A. Grzybowski, Nat. Commun. 10, 1 (2019).

54 C. A. Grambow, Y.-P. Li, and W. H. Green, J. Phys. Chem. A 123, 5826 (2019).

55 Y.-P. Li, K. Han, C. A. Grambow, and W. H. Green, J. Phys. Chem. A 123, 2142 (2019).

56 M. E. Fortunato, C. W. Coley, B. C. Barnes, and K. F. Jensen, J. Chem. Inf. Model. 60, 3398 (2020).

57 (accessed: 04/2021), https://github.com/rxn4chemistry/rxnfp.

58 D. Rogers and M. Hahn, J. Chem. Inf. Model. 50, 742 (2010).

59 G. Landrum, "Rdkit: Open-source cheminformatics," (2006), https://www.rdkit.org/.

60 Y. Guan, C. W. Coley, H. Wu, D. Ranasinghe, E. Heid, T. J. Struble, L. Pattanaik, W. H. Green, and K. F. Jensen, Chem. Sci. 12, 2198 (2021). 